Massachusetts. They found that the first sighting of a migratory bird, although one of the most commonly used statistics, can be confounded by population size. As bird numbers decline, there are fewer early outliers, making the first birds harder to spot. By extension, growing populations are expected to distort results in the opposite direction.

Instead, the team used the mean arrival date, which did not change with population size. Overall, the birds' mean arrival was two and a half days earlier by the end of the period covered by the data set than at the start.

\section{ANIMAL BEHAVIOUR}

\section{Crowd control}

Am. J. Primatol. doi:10.1002/ajp.20601 (2008)

Many researchers would expect parasitic infection rates to increase as groups of animals get bigger and more hosts are available. Contrary to this, researchers reveal that as groups of red colobus monkeys (Procolobus rufomitratus) get larger, they have fewer parasites.

Tamaini Snaith at McGill University in Montreal, Canada, and her colleagues made the discovery while studying the monkeys in Uganda. They tested faeces for parasites and monitored group dynamics. The researchers noticed that large groups tended to spread out more than smaller ones, and suggest that this could lower infection rates.

They add that although the monkeys may be spreading out in response to the parasites, feeding competition could also be driving the spread, making the parasite reduction a happy accident.

\section{IMMUNOLOGY}

\section{The power of tick spit}

J. Exp. Med. doi:10.1084/jem.20072689 (2008)

Proteins used by ticks to evade their hosts' immune systems may one day provide a new way to fight inflammation, researchers say.

Compounds in tick saliva block inflammation and allow the bloodsucking parasites to feed off a host for long time periods without alerting its immune system. Amanda Proudfoot and Christine Power of Merck Serono Geneva Research Centre in Switzerland and their team isolated a family of tick saliva proteins that very selectively bind specific pro-inflammatory members of a protein family called chemokines.

Two of these proteins, which have been named evasins, produced no obvious allergic reaction and reduced inflammation when given to mice suffering from several conditions, including skin or joint inflammation and lung injury.

\section{BOTANY \\ Biofuel heir apparent?}

Global Change Biol. 14, 2000-2014 (2008)

The perennial grass Miscanthus $\times$ giganteus (pictured below) is photosynthetic royalty. In field tests conducted by Stephen Long and his co-workers at the University of Illinois in Urbana, this sterile hybrid converted $1 \%$ of solar energy into biomass that could be harvested to make cellulosic ethanol. That's ten times the standard $0.1 \%$ efficiency cited for plants in general.

The team compared Miscanthus with switchgrass (Panicum virgatum), a current US favourite for the cellulosic fuel of the future. Miscanthus produced an average of 30 tonnes of harvestable biomass per hectare; switchgrass produced just 10 tonnes.

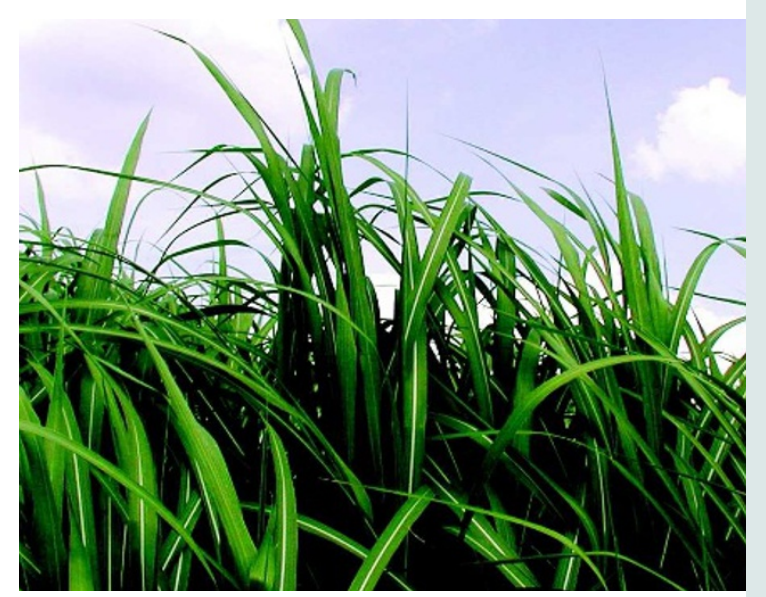

BIOPHYSICS

\section{Mob rule}

Proc. Natl Acad. Sci. USA 105, 11754-11759 (2008)

The crowding inherent within cells may affect not only protein movement and folding, but also shape, according to Pernilla Wittung-Stafshede at Rice University in Houston, Texas, and her colleagues.

They focused on the VlsE protein, a proposed virulence factor in Borrelia burgdorferi, the bacterium that causes Lyme disease. VlsE is usually rugby-ball shaped, but the team found that it adopts different equilibrium shapes in vitro in the presence of varying levels of a polymeric 'crowding agent' that mimics cytoplasmic macromolecules. When the native protein is loosened up by a denaturing agent or by heat, two new structures - a 'bean' shape and a roughly spherical conformation - intervene between the rugby ball and the denatured protein as soon as the crowding agent is added.

If crowding can be 'tuned', it might be possible to expose different sites in proteins and alter their behaviour.

\section{JOURNAL CLUB}

\author{
Michelle D. Wang \\ Howard Hughes Medical \\ Institute, Cornell University, \\ Ithaca, New York
}

\section{A biophysicist marvels at the idea of grabbing microscopic particles with light by tweaking its phase.}

Light carries energy and momentum. Have you ever gazed at a comet on a hot summer night? The dust tail seen streaming out from a comet is caused by sunlight bombarding dust particles from its surface and pushing them away from the Sun. The same radiation pressure can be used to 'trap', or hold, microscopic particles. And if an item of interest - for example, a biological molecule - is attached to a particle subject to trapping, it can then be manipulated as the trap is moved.

So how does one generate optical traps? Conventionally, a laser beam is directed through the objective lens of a microscope and focused to a small spot very close to the specimen. The trapping force relies on the gradient of the laser's intensity - the tighter the focus, the greater the intensity change within the focused beam, and the greater the trapping force.

For a long time, this has been the only type of trap available. But not any more! David Grier and his colleagues have created a new type of trap that relies on the gradient of the 'phase' of a laser's light as well as its intensity (Y. Roichman et al. Phys. Rev. Lett. 100, 013602 ; 2008). Light waves, like ocean waves, have crests and troughs. The phase of a light wave specifies what position within the wave, from crest to trough, the light is in at a given moment. By tweaking the phase of the laser in the trap, the researchers are able not only to hold a particle steady, but also to move it in a line or spin it around in a circle. It is now possible to design optical traps that are more flexible and versatile, and that can generate as much trapping force as before, but with less light.

I would not be surprised if these traps soon become one of the must-have tools in single-molecule biophysics, cell biology and colloidal physics.

Discuss this paper at http://blogs. nature.com/nature/journalclub 